Volume 70, Number 1, Pages 82-99(2021)

DOI: $10.31801 /$ cfsuasmas.736132

ISSN 1303-5991 E-ISSN 2618-6470

Received by the editors: May 13, 2020; Accepted: October 07, 2020

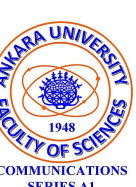

\title{
STATISTICAL EXTENSION OF BOUNDED SEQUENCE SPACE
}

\author{
Maya ALTINOK ${ }^{1}$, Mehmet KÜÇÜKASLAN ${ }^{2}$, and Umutcan KAYA ${ }^{2}$ \\ ${ }^{1}$ Department of Natural and Mathematical Sciences, Tarsus University, Mersin, Turkey \\ ${ }^{2}$ Department of Mathematics, Mersin University, Mersin, Turkey
}

\begin{abstract}
In this paper by using natural density real valued bounded sequence space $l_{\infty}$ is extented and statistical bounded sequence space $l_{\infty}^{s t}$ is obtained. Besides the main properties of the space $l_{\infty}^{s t}$, it is shown that $l_{\infty}^{s t}$ is a Banach space with a norm produced with the help of density. Also, it is shown that there is no matrix extension of the space $l_{\infty}$ that its bounded sequences space covers $l_{\infty}^{s t}$. Finally, it is shown that the space $l_{\infty}$ is a non-porous subset of $l_{\infty}^{s t}$.
\end{abstract}

\section{INTRODUCTION}

The notion of convergence is one of the main tools of mathematical analysis to understand the geometric and algebraic structures of the space. For example, in order to determine whether a space is complete, it is necessary and sufficient to check whether Cauchy sequences of the space are convergent. There are a lot of generalization of classical convergence such as Cesàro, Nörlund, Riesz, Abel, etc. Most popular of them is statistical convergence, defined in 1951 by authors Fast 10 and Steinhaus 24 independently, whose roots are based on the work of Zygmund 25. It has been extensively studied by many researchers for the last ten decades. Statistical convergence is applied different fields of mathematics: approximation theory 13], measure theory [19], 7], probability theory [22, metric spaces [5], 6], 12], 16, 20], number theory 9], 14], summability theory [1, 8], [18], 21], 23], etc. Almost all these studies have yielded many excellent results using either natural density or any generalization of natural density.

2020 Mathematics Subject Classification. 40A05, 26A03, MB05.

Keywords and phrases. Natural density, statistical supremum, sequence spaces.

mayaaltinok@tarsus.edu.tr; mkkaslan@gmail.com-Corresponding author; umutcanmath@gmail.com

(D) 0000-0002-6671-743X; 0000-0002-3183-3123; 0000-0002-0419-6106. 
Recall the definition of natural density: Natural density of $A \subseteq \mathbb{N}$ is defined as follows if the limit exists

$$
\delta(A):=\lim _{n \rightarrow \infty} \frac{\operatorname{card}(A(n))}{n}
$$

where

$$
A(n)=\{k \leq n: k \in \mathbb{N}\}=A \cap\{1,2, \ldots, n\} .
$$

If a sequence $\tilde{x}=\left(x_{n}\right)$ has a $P$-property except a subset of natural numbers with density zero, then we say that $\tilde{x}=\left(x_{n}\right)$ has the $P$-property for almost all $n$, and we use (a.a.n) for brevity.

Let, $\tilde{x}=\left(x_{n}\right)$ be a real valued sequence and $l \in \mathbb{R}$. If for every $\varepsilon>0$

$$
\delta(A(\varepsilon)(n))=0
$$

where

$$
A(\varepsilon)=\left\{n:\left|x_{n}-l\right| \geq \varepsilon\right\}
$$

then $\tilde{x}=\left(x_{n}\right)$ is called statistical convergent to $l$. It is denoted by $s t-\lim x_{n}=l$.

The set of statistical convergent sequences is denoted by $c_{s t}$ :

$$
c_{s t}:=\left\{\tilde{x}=\left(x_{n}\right): \exists l \in \mathbb{R} \text { such that } s t-\lim x_{n}=l\right\} .
$$

If $l=0$, then we are going to use $c_{s t}^{0}$. So, it is clear from (1) and (2) that $c^{0} \subset c_{s t}^{0} \subset c_{s t}$ and $c \subset c_{s t}$ hold.

Let $\tilde{x}=\left(x_{n}\right)$ be a sequence. If for every $\varepsilon>0$, there exist $n_{0}(\varepsilon) \in \mathbb{N}$ such that

$$
\delta\left(\left\{n \in \mathbb{N}:\left|x_{n}-x_{n_{0}}\right| \geq \varepsilon\right\}\right)=0
$$

holds, then the sequence $\tilde{x}=\left(x_{n}\right)$ is called statistical Cauchy sequence. The set of all statistical Cauchy sequences is denoted by $\mathcal{C}_{s t}$.

In [11, Fridy gave the following important Theorem which gives the relation between statistical convergence and classical convergence, $c$ and $c_{s t}$. In terms of showing this relationship, it can be considered as the basic result of this theory.

Theorem 1. The following statements are equivalent:

(1) $\tilde{x}$ is statistical convergent sequence;

(2) $\tilde{x}$ is statistical Cauchy sequence;

(3) $\tilde{x}$ is a sequence for which there is a convergent sequence $\tilde{y}$ such that $x_{n}=y_{n}$ (a.a.n).

As a result of Theorem 1 1 , the relationship between classical Cauchy sequence and statistical Cauchy sequences can be given as follows:

Remark 2. $\tilde{x}=\left(x_{n}\right)$ is statistical Cauchy sequence if and only if there exists a Cauchy sequence $\tilde{y}=\left(y_{n}\right)$ such that $x_{n}=y_{n}$ (a.a.n).

It is well known from the general theory that convergent and Cauchy sequences are member of bounded sequences space $l_{\infty}$ where

$$
l_{\infty}:=\left\{\tilde{x}=\left(x_{n}\right): \sup _{n}\left|x_{n}\right|<\infty\right\} .
$$


Unfortunately, this general expression is not true for statistical convergence and statistical Cauchy sequences even if they have a convergent and Cauchy subsequences, respectively 11].

To see this let us consider following sequences $\tilde{x}=\left(x_{n}\right)$ and $\tilde{y}=\left(y_{n}\right)$ defined as:

$$
x_{n}= \begin{cases}n, & n \in \mathbb{P}(=\text { prime numbers }) \\ 1, & n \notin \mathbb{P},\end{cases}
$$

and

$$
y_{n}= \begin{cases}3, & n \in \mathbb{P}(=\text { prime numbers }) \\ 1, & n \notin \mathbb{P} .\end{cases}
$$

The sequence $\tilde{x}=\left(x_{n}\right)$ is an statistical convergent (and statistical Cauchy) sequence but it is not convergence (and Cauchy) sequence. Also, the sequence $\tilde{y}=\left(y_{n}\right)$ is statistical convergent (and Cauchy) but not convergent (and Cauchy). Clearly, $\tilde{x}=\left(x_{n}\right) \notin l_{\infty}$ and $\tilde{y}=\left(y_{n}\right) \in l_{\infty}$.

So, it can be say that, the set of statistical convergent (or statistical Cauchy) sequences is whether a subset of $l_{\infty}$ or not include $l_{\infty}$.

Therefore, as in the classical case it should be found a sequence space looks like $l_{\infty}$ which contains $c_{s t}$ and $\mathcal{C}_{s t}$.

Basically, to eliminate this deficiency is the main purpose of this work. To overcome this deficiency a new sequence space will be built and its properties will be investigated.

\section{Statistical Bounded Sequnce Space $l_{\infty}^{s t}$}

In this section, the concept of statistical boundedness will be defined by using natural density as a generalization of the classical boundedness of sequences, and a new sequence space $l_{\infty}^{s t}$ which is called statistically bounded sequences will be created.

Definition 3. [14](Statistical boundedness) Let $\tilde{x}=\left(x_{n}\right)$ be a sequence of real numbers. If there exists $M>0$ such that

$$
\delta\left(\left\{n:\left|x_{n}\right| \geq M\right\}\right)=0,\left(\text { or } \delta\left(\left\{n:\left|x_{n}\right|<M\right\}\right)=1\right)
$$

holds, then, $\tilde{x}$ is called statistical bounded sequence.

The set of all statistical bounded sequences is denoted by $l_{\infty}^{s t}$ :

$$
l_{\infty}^{s t}:=\left\{\tilde{x}=\left(x_{n}\right): \exists M>0 \text { such that } \delta\left(\left\{n:\left|x_{n}\right| \geq M\right\}\right)=0\right\} .
$$

Remark 4. If $\tilde{x}=\left(x_{n}\right)$ is a bounded sequence then $\tilde{x}$ is a statistical bounded sequence. That is; $l_{\infty} \subseteq l_{\infty}^{s t}$.

Proof. Since $\tilde{x}=\left(x_{n}\right)$ is a bounded, then there exists $M>0$ such that $\left|x_{n}\right| \leq M$ holds for all $n \in \mathbb{N}$. This implies that

$$
\left\{n:\left|x_{n}\right| \geq M\right\}=\emptyset \text {. }
$$


So,

$$
\delta\left(\left\{n:\left|x_{n}\right| \geq M\right\}\right)=0 .
$$

Therefore, this gives that $\tilde{x} \in l_{\infty}^{s t}$.

Let's consider an example below to see that the inclusion given above is strict.

Example 5. Let $\tilde{x}=\left(x_{n}\right)$ defined as follows:

$$
x_{n}= \begin{cases}k, & n=k^{2} \\ (-1)^{n}, & n \neq k^{2}\end{cases}
$$

for all $n \in \mathbb{N}$.

It is clear for $M=2$ that

$$
\left\{n:\left|x_{n}\right| \geq 2\right\}=\left\{n: n=k^{2}, k \in \mathbb{N}\right\}
$$

holds.

Since $\delta\left(\left\{k^{2}: k \in \mathbb{N}\right\}\right)=0$, then the sequence $\tilde{x}=\left(x_{n}\right)$ is statistical bounded but it is not bounded.

Following Lemma will be show that the space $l_{\infty}^{s t}$ is not cover the space $s$ where $s$ denotes the set of all real valued sequences.

Lemma 6. $s \backslash l_{\infty}^{s t} \neq \emptyset$.

Proof. Actually, let $\tilde{x}=\left(x_{n}\right)$ defined as follows:

$$
x_{n}:= \begin{cases}\sqrt{n}, & n=k^{2} \\ (-1)^{n} n^{2}, & n \neq k^{2} .\end{cases}
$$

Since $\left\{n:\left|x_{n}\right| \geq M\right\}=\mathbb{N}-A$, where $A$ is finite subset of $\mathbb{N}$, then

$$
\delta\left(\left\{n:\left|x_{n}\right| \geq M\right\}\right)=1 .
$$

So, the sequence $\tilde{x}$ is not statistical bounded.

Theorem 7. The cardinality of the set $s \backslash l_{\infty}^{s t}$ is $c$ (=continuum).

Proof. As a result of Lemma 6, we know that the set $s \backslash l_{\infty}^{s t}$ has at least one element. Let's see that it contains more than one element. For a fixed real number $r \in[0,1]$, let us construct a sequence $b(r)=\left(b_{n}(r)\right)_{n \in \mathbb{N}}$ as follows:

$$
b_{n}(r)= \begin{cases}n^{r}, & n \in 3 \mathbb{N} \\ 1, & n \in \mathbb{N}-3 \mathbb{N}\end{cases}
$$

The sequence $b_{n}^{r} \notin l_{\infty}^{s t}$ for all $r \in[1, \infty)$. So, we can define a function $f$ as follows,

$$
f:[0,1] \rightarrow s \backslash_{\infty}^{s t}
$$

such that

$$
f(r):=b(r) .
$$

It is clear that the function $f$ is an injection between $[0,1]$ and $s \backslash_{\infty}^{s t}$. So, cardinality of $s \backslash l_{\infty}^{s t}$ is bigger than cardinality of the interval $[0,1]$. Therefore, the cardinality of $s \backslash_{\infty}^{s t}$ is continuum. 
Theorem 8. Every statistical convergent (or statistical Cauchy) sequence is also statistical bounded sequence. That is, $c_{s t} \subset l_{\infty}^{s t}$ and $\mathcal{C}_{s t} \subset l_{\infty}^{s t}$ holds. Converses of this inclusions are not true, in general.

Proof. Let $\tilde{x}=\left(x_{n}\right)$ be a statistical convergent sequence to $x_{0}$. So, from (2)

$$
\delta(A(\varepsilon))=\delta\left(\left\{n \in \mathbb{N}:\left|x_{n}-x_{0}\right| \geq \varepsilon\right\}\right)=0
$$

for every $\varepsilon>0$.

So, for any sufficiently large $M>0$, we have

$$
\left\{n \in \mathbb{N}:\left|x_{n}\right| \geq M\right\} \subset\left\{n \in \mathbb{N}:\left|x_{n}-x_{0}\right| \geq M\right\} \subset\left\{n \in \mathbb{N}:\left|x_{n}-x_{0}\right| \geq \varepsilon\right\} .
$$

This inclusion implies that

$$
\delta\left(\left\{n \in \mathbb{N}:\left|x_{n}\right| \geq M\right\}\right)=0 .
$$

Thus, $\tilde{x}=\left(x_{n}\right)$ is a statistical bounded sequence.

If $\tilde{x}=\left(x_{n}\right)$ statistical Cauchy then from Theorem 1 the sequence $\tilde{x}=\left(x_{n}\right)$ is also statistical convergent. So, it is statistical bounded. For the converse of theorem, let $\tilde{x}=\left(x_{n}\right)$ as follows:

$$
x_{n}= \begin{cases}k^{2}, & n=k^{2}, \\ 1, & n=k^{2}+1, \\ 2, & n=k^{2}+2, \\ 0, & \text { otherwise. }\end{cases}
$$

It is clear that the sequence $\tilde{x}=\left(x_{n}\right) \in l_{\infty}^{s t}$ but its not statistical convergent or statistical Cauchy sequence.

Corollary 9. Every convergent sequence is also statistical bounded sequence.

Lemma 10. Let $K \subset \mathbb{N}$ with $\delta(K)=1$. Then, there exists a subset $S \subseteq K$ with $\delta(S)=1$ such that for an increasing sequence of natural numbers $\left(n_{k}\right)$ :

$$
S=\left\{\left(n_{k}\right)_{k \in \mathbb{N}}: k \in \mathbb{N}\right\} .
$$

Proof. Let's denote the set $\mathbb{N}-K$ by $U$. Since $\delta(K)=1$, then it is clear that $\delta(U)=0$.

(i) If $U=\emptyset$, the claim is clear, for $S=\mathbb{N}$ and $n_{k}=k$ for all $k \in \mathbb{N}$.

(ii) If $U \neq \emptyset$ and $U$ is finite, then the claim is true for $S=\left\{n+u_{\max }: n \in \mathbb{N}\right\}$, where $u_{\max }$ is the biggest element of $\mathrm{U}$.

(iii) If $U \neq \emptyset$ and $U$ is denumerable, let us denote the m-th element of the set $U$ with $u_{m}$ for $m \in \mathbb{N}$.

Construct some sets of natural numbers as follows:

$$
A_{m}=\left\{k \in K: u_{m}<k<u_{m+1}\right\}
$$

for all $m \in \mathbb{N}$. It is clear that $K=\bigcup_{m \in \mathbb{N}} A_{m}$. We hasten to add that for some $m \in \mathbb{N}$ the $A_{m}$ 's may be an empty set. This is not problem for the claim. Now, 
put the sequence $\left(n_{k}\right)_{k \in \mathbb{N}}$ as follows:

$$
n_{k}=\min \left(\bigcup_{m \in \mathbb{N}} A_{m} \backslash \bigcup_{i \in I_{k-1}} n_{i}\right),
$$

where $I_{0}=\emptyset$ and $I_{k}=\{1,2, \ldots, k\}$.

Let $S=\left\{n_{k}: k \in \mathbb{N}\right\}$. This tells us, the set $S$ consists consecutive blocks of natural numbers of any finite length. Now, from construction of $A_{m}$ 's the set $S$ also consists of all removed elements of the set $U$ (which has density zero) from the set $K$. Therefore, $\delta(S)=1$ and monotonicity of $\left(n_{k}\right)$ is clear from the construction.

Theorem 11. If $\tilde{x}=\left(x_{n}\right) \in l_{\infty}^{s t}$, then there exists a subset $S$ of $\mathbb{N}$ with $\delta(S)=1$ such that $\left(x_{n_{k}}\right)_{n_{k} \in S} \in l_{\infty}$ holds.

Proof. From Definition 3 we know that the set

$$
\left\{k \in \mathbb{N}:\left|x_{k}\right| \geq M\right\}
$$

has zero density for some $M>0$. Put $K=\left\{k \in \mathbb{N}:\left|x_{k}\right|<M\right\}$. Clearly $\delta(K)=1$. From Lemma 4 take the nonempty subset $S$ of $K$ such that

$$
S=\left\{\left(n_{k}\right)_{n_{k} \in \mathbb{N}}: n_{k} \in \mathbb{N}\right\}
$$

where the sequence $\left(n_{k}\right)_{k \in \mathbb{N}}$ is an increasing sequence of natural numbers.

It is clear that, the subsequence $\tilde{x}=\left(x_{n_{k}}\right)_{n_{k} \in \mathbb{S}}$ of sequence $\left(x_{n}\right)_{n \in \mathbb{N}}$ is an element of $l_{\infty}$.

Theorem 12 (Decomposition theorem). If $\tilde{x}=\left(x_{n}\right) \in{ }_{\infty}^{s t} \backslash l_{\infty}$, then there exist $\tilde{y}=\left(y_{n}\right) \in_{\infty}$ and $\tilde{z}=\left(z_{n}\right) \notin l_{\infty}$ such that

$$
x_{n}:=y_{n}+z_{n}
$$

holds for all $n \in \mathbb{N}$. However, this decomposition is not unique.

Proof. Since $\tilde{x}=\left(x_{n}\right) \in l_{\infty}^{s t}$, then there exists $M>0$ such that

$$
\delta(K)=\delta\left(\left\{n:\left|x_{n}\right|<M\right\}\right)=1 .
$$

Therefore, it is clear from Theorem 11 that there exists a monotone increasing sequence of natural numbers $\left(n_{k}\right)_{k \in \mathbb{N}}$ such that

$$
\delta\left(\left\{n_{k}: k \in \mathbb{N}\right\}\right)=1 .
$$

Hence, desired sequences $\tilde{y}=\left(y_{n}\right)$ and $\tilde{z}=\left(z_{n}\right)$ can be defined as follows

$$
y_{n}= \begin{cases}x_{n_{k}}, & n=n_{k}, \\ 0, & n \neq n_{k},\end{cases}
$$

and

$$
z_{n}= \begin{cases}x_{n}, & n \neq n_{k}, \\ 0, & n=n_{k} .\end{cases}
$$

That is, following two sequences are created from the sequence $\tilde{x}=\left(x_{n}\right)$ :

$$
\left(y_{n}\right)=\left(0, \ldots, 0, x_{n_{1}}, 0, \ldots, 0, x_{n_{k}}, 0, \ldots\right)
$$




$$
\left(z_{n}\right)=\left(x_{1}, \ldots, x_{n_{1}-1}, 0, x_{n_{1}+1}, \ldots, x_{n_{k}-1}, 0, x_{n_{k}+1}, \ldots\right)
$$

So, $x_{n}=y_{n}+z_{n}$ holds for all $n \in \mathbb{N}$.

\section{Statistical sup-norm and Associated Norm Space $l_{\infty}^{s t}$}

By considering the set of statistical upper bound of the sequence with the help of natural density, statistical sup-norm will be defined in this section (See for more information in [2], [3] and [4]).

In addition to the main properties of the set $l_{\infty}^{s t}$, it will be shown that this set is a statistical Banach space according to the statistical sup-norm.

For any sequence $\tilde{x}=\left(x_{n}\right)$, let us denote its absolute value $|\tilde{x}|$ such that

$$
|\tilde{x}|:=\left(\left|x_{n}\right|\right)_{n \in \mathbb{N}}=\left(\left|x_{1}\right|,\left|x_{2}\right|, \ldots,\left|x_{n}\right|, \ldots\right) .
$$

Now, let us define a norm over $l_{\infty}^{s t}$ by the help of density.

Definition 13. [15] (i)(Statistical upper bound) A number $m \in \mathbb{R}$ is called statistical upper bound of $\tilde{x}=\left(x_{n}\right)$ if

$$
\delta\left(\left\{k: x_{k}<m\right\}\right)=1,\left(\text { or } \delta\left(\left\{k: x_{k} \geq m\right\}\right)=0\right)
$$

holds. The set of all statistical upper bound of $\tilde{x}=\left(x_{n}\right)$ is denoted by $U^{\text {st }}(\tilde{x})$ such that

$$
U^{s t}(\tilde{x}):=\left\{m \in \mathbb{R}: \delta\left(\left\{k: x_{k} \geq m\right\}\right)=0\right\}
$$

(ii) (Statistical Supremum) A number $l \in \mathbb{R}$ is called statistical supremum of $\tilde{x}=\left(x_{n}\right)$ if $l$ is the infimum of $U^{\text {st }}(|\tilde{x}|)$. That is;

$$
s t-\sup _{n} x_{n}:=\inf _{m}\left\{m: m \in U^{s t}(|\tilde{x}|)\right\} .
$$

Lemma 14. For any given sequence $\tilde{x}=\left(x_{n}\right)_{n \in \mathbb{N}}$, the set $U^{\text {st }}(\tilde{x})$ is closed subset of $\mathbb{R}$.

Proof. Let us denote the closure of the set $U^{s t}(\tilde{x})$ by $\bar{U}^{s t}(\tilde{x})$. It is clear that

$$
U^{s t}(\tilde{x}) \subset \bar{U}^{s t}(\tilde{x})
$$

holds trivially. Now, let $m \in \bar{U}^{s t}(\tilde{x})$ be an arbitrary element and assume that $m \notin U^{s t}(\tilde{x})$. From the definition of closure point, for every $\varepsilon>0$ we have,

$$
B_{e}(m, \varepsilon) \cap U^{s t}(\tilde{x}) \neq \emptyset,
$$

where $B_{e}(m, \varepsilon)$ denotes the ball centered $m$ with radius $\varepsilon$ in the Euclidean metric $e$.

Let $l \in \bar{U}^{s t}(\tilde{x})$ such that (3) is satisfied. Since $m \notin U^{s t}(\tilde{x})$, then $l$ is not less than or equal to $m$. Otherwise, from the result in 15 that $m \in U^{s t}(\tilde{x})$ must be holds, which contradicts to our assumption. Hence, $l>m$.

Now, without loss of generality assume that $l$ is the least real number which belongs to $B_{e}(m, \varepsilon)$ satisfying (3). 
If we choose $\varepsilon:=\frac{l-m}{2}>0$, then

$$
B_{e}(m, \varepsilon) \cap U^{s t}(\tilde{x})=\emptyset .
$$

This is a contradiction $m \in \bar{U}^{s t}(\tilde{x})$. Therefore, $m \in U^{s t}(\tilde{x})$.

Denote the number $\inf \left\{m: m \in U^{s t}(|\tilde{x}|)\right\}$ by $\|\tilde{x}\|_{\infty}^{s t}$. It is clear from the Lemma 14 that

is well defined function.

$$
\|.\|_{\infty}^{s t}: l_{\infty}^{s t} \rightarrow[0, \infty)
$$

Lemma 15. If $\tilde{x}=(0,0, \ldots)$, then $\|\tilde{x}\|_{\infty}^{s t}=0$ holds. The converse is not true.

Proof. If $\tilde{x}=(0,0, \ldots)$ then $U^{s t}(|\tilde{x}|)=[0, \infty)$. So, $\|\tilde{x}\|_{\infty}^{s t}=0$ is trivial.

For converse, let a sequence $\tilde{x}=\left(x_{n}\right)$ as

$$
x_{n}:=\left\{\begin{array}{ll}
k, & n=k^{2} \\
0, & n \neq k^{2}
\end{array},\right.
$$

The sequence $\tilde{x}=\left(x_{n}\right)$ belongs to $l_{\infty}^{s t}$ and $U^{s t}(|\tilde{x}|)=[0, \infty)$. This gives that

$$
\|\tilde{x}\|_{\infty}^{s t}=0 .
$$

But $\tilde{x} \neq(0,0, \ldots)$.

Lemma 16. $U^{\text {st }}(|\lambda \tilde{x}|)=|\lambda| \cdot U^{\text {st }}(|\tilde{x}|)$ holds for any $\lambda \in \mathbb{R}$.

Proof. Let $m \in U^{s t}(|x|)$ be an arbitrary element. Then, we have $\delta\left(\left\{n:\left|x_{n}\right|>\right.\right.$ $m\})=0$. Assume that $|\lambda| . m \notin U^{s t}(|\lambda \tilde{x}|)$. That is,

$$
\delta\left(\left\{n:\left|\lambda x_{n}\right|>|\lambda| m\right\}\right)>0
$$

holds. Since, the following equality

$$
\left\{n:|\lambda|\left|x_{n}\right|>|\lambda| \cdot m\right\}=\left\{n:\left|x_{n}\right|>m\right\}
$$

holds, then $\delta\left(\left\{n:\left|x_{n}\right|>m\right\}\right)>0$. This is a contradiction.

The converse equality can be obtained in a similar way. So it is omitted here.

Corollary 17. For any $\lambda \in \mathbb{R}$, we have

$$
\|\lambda . x\|_{\infty}^{s t}=|\lambda| .|| x \|_{\infty}^{s t} .
$$

Lemma 18. If $l_{1} \in U^{s t}(|\tilde{x}|)$ and $l_{2} \in U^{s t}(|\tilde{y}|)$, then $l_{1}+l_{2} \in U^{s t}(|\tilde{x}+\tilde{y}|)$.

Proof. Let $l_{1} \in U^{s t}(\tilde{x})$ and $l_{2} \in U^{s t}(\tilde{y})$ be any arbitrary elements. Therefore, we have

and

$$
\delta\left(\left\{n:\left|x_{n}\right|>l_{1}\right\}\right)=0
$$

$$
\delta\left(\left\{n:\left|y_{n}\right|>l_{2}\right\}\right)=0 .
$$

Assume that $l_{1}+l_{2} \notin U^{s t}(|\tilde{x}+\tilde{y}|)$.

Since the following inclusion

$$
\left\{n:\left|x_{n}+y_{n}\right| \geq l_{1}+l_{2}\right\} \subset
$$




$$
\subset\left\{n:\left|x_{n}\right|+\left|y_{n}\right| \geq l_{1}+l_{2}\right\} \subset\left\{n:\left|x_{n}\right| \geq l_{1}\right\} \cup\left\{n:\left|y_{n}\right| \geq l_{2}\right\}
$$

holds, then we have

$$
\frac{\left|\left\{n:\left|x_{n}+y_{n}\right| \geq l_{1}+l_{2}\right\}\right|}{n} \leq \frac{\left|\left\{n:\left|x_{n}\right| \geq l_{1}\right\}\right|}{n}+\frac{\left|\left\{n:\left|y_{n}\right| \geq l_{2}\right\}\right|}{n} .
$$

Limit of left hand side in the above inequality is not zero when $n$ tends to infinity and this gives that in the right hand side at least $l_{1} \notin U^{s t}(|\tilde{x}|)$ or $l_{2} \notin U^{s t}(|\tilde{y}|)$ must be hold. This is contradiction to the hypothesis.

Corollary 19. For any sequences $\tilde{x}, \tilde{y} \in l_{\infty}^{s t}$,

$$
\|\tilde{x}+\tilde{y}\|_{\infty}^{s t} \leq\|\tilde{x}\|_{\infty}^{s t}+\|\tilde{y}\|_{\infty}^{s t}
$$

holds.

Theorem 20. (i) The space $l_{\infty}^{\text {st }}$ is a linear space over $\mathbb{R}$.

(ii) $\|.\|_{\infty}^{s t}$ is a pseudo norm on the space $l_{\infty}^{\text {st }}$.

Proof. From Lemma 15, Lemma 16 and Lemma 18, proof can be obtained.

Theorem 21. $l_{\infty}^{s t}$ is not seperable vector space.

Proof. It is well known that, all subset of seperable space are seperable. Since $l_{\infty}$ is not seperable and $l_{\infty} \subsetneq l_{\infty}^{s t}$, then $l_{\infty}^{s t}$ is not seperable space.

Let us define a relation " $\sim$ " on the pace $l_{\infty}^{s t}$ as:

For $\tilde{x}, \tilde{y} \in l_{\infty}^{s t}$,

$$
\text { " } \tilde{x} \sim \tilde{y} \Longleftrightarrow\|\tilde{x}-\tilde{y}\|_{\infty}^{s t}=0 . "
$$

It is clear that $" \sim "$ is an equivalence relation on the space $l_{\infty}^{s t}$. So, we have quotient space

$$
l_{\infty}^{s t} / \sim:=\left\{[\tilde{x}]: \tilde{x} \in l_{\infty}^{s t}\right\}
$$

where $[\tilde{x}]$ is the equivalence class of the sequence $\tilde{x}$,

$$
[\tilde{x}]:=\left\{\tilde{y} \in l_{\infty}^{s t}: \tilde{x} \sim \tilde{y}\right\} .
$$

The relation $\sim$ is also an equivalence relation on the space $l_{\infty}$ and $\left(l_{\infty} / \sim\right)$ denotes the set of all equivalence classes of $l_{\infty}$.

For simplicity, let us denote the quotient spaces $\left(l_{\infty}^{s t} / \sim\right)$ and $\left(l_{\infty} / \sim\right)$ by $\tilde{l}_{\infty}^{s t}$ and $\tilde{l}_{\infty}$, respectively. Namely, we have following quotient spaces:

$$
\begin{aligned}
& \tilde{l}_{\infty}:=l_{\infty} / \sim:=\left\{[\bar{x}]:\left(\bar{x}_{n}\right)=\left(\bar{x}_{1}, \bar{x}_{2}, \ldots, \bar{x}_{n}, \ldots\right) \in l_{\infty}\right\}, \\
& \tilde{l}_{\infty}^{s t}:=l_{\infty}^{s t} / \sim:=\left\{[\tilde{x}]:\left(\tilde{x}_{n}\right)=\left(x_{1}, x_{2}, \ldots, x_{n}, \ldots\right) \in l_{\infty}^{s t}\right\} .
\end{aligned}
$$

Theorem 22. $\left(\tilde{l}_{\infty}^{s t},\|\cdot\|_{\infty}^{s t}\right)$ is a normed space.

Lemma 23. Let $\tilde{x}=\left(x_{n}\right)$ and $\tilde{y}=\left(y_{n}\right) \in l_{\infty}^{s t}$ and $A=\left\{n: x_{n} \neq y_{n}\right\}$. Then $\delta(A)=0$ if and only if $\tilde{y} \in[\tilde{x}]$. 
Proof. Since $\delta(A)=0$, then for every arbitrary $\varepsilon>0$,

$$
\delta\left(\left\{n:\left|x_{n}-y_{n}\right| \geq \varepsilon\right\}\right)=0
$$

holds. It means that $\varepsilon \in U^{s t}(|\tilde{x}-\tilde{y}|)$. Therefore,

$$
\left\|x_{n}-y_{n}\right\|_{\infty}^{s t}=0
$$

satisfied. This implies that $\tilde{x} \sim \tilde{y}$. So, $\tilde{y} \in[\tilde{x}]$. Converse is obtained from the definition of statistical-sup norm.

Lemma 24. For all $[\bar{x}] \in \tilde{l}_{\infty}$, there exists a $\tilde{x} \in l_{\infty}^{\text {st }}$ such that $[\bar{x}] \subset[\tilde{x}]$.

Proof. Let $[\bar{x}] \in \tilde{l}_{\infty}$ be an arbitrary equivalence class of sequences. Consider an associate sequence $\tilde{x}=\left(x_{n}\right)$ in $l_{\infty}^{s t}$ as:

$$
x_{n}= \begin{cases}k, & n=k^{2}, \\ \bar{x}_{n}, & n \neq k^{2} .\end{cases}
$$

It is clear that $A:=\left\{n: x_{n} \neq \bar{x}_{n}\right\}=\left\{n=k^{2}: k \in \mathbb{N}\right\}$ and asymptotic density of this set is zero. Hence, $\bar{x} \sim \tilde{x}$. So, from Lemma 23 , we have $\bar{x} \in[\tilde{x}]$.

Now, let $\bar{y}=\left(\bar{y}_{n}\right) \in[\bar{x}]$ be an arbitrary sequence such that the set $B=\{n$ : $\left.\bar{x}_{n} \neq \bar{y}_{n}\right\}$ has natural density zero. If we consider $C=\left\{n: x_{n} \neq \bar{y}_{n}\right\}$ then $C=A \cap B$ holds. This immediately gives that $\delta(C)=0$. That is, $\bar{y} \sim \tilde{x}$ satisfied and $[\bar{x}] \subset[\tilde{x}]$.

Lemma 25. Let $\tilde{x}=\left(x_{n}\right)$ and $\tilde{y}=\left(y_{n}\right) \in l_{\infty}^{\text {st }}$ be arbitrary elements. Then, $\tilde{y} \in[\tilde{x}]$ if and only if $\left\|x_{n}-y_{n}\right\|_{\infty}^{s t}=0$.

Proof. Let $\tilde{y} \in[\tilde{x}]$ be an arbitrary sequence. Then, since $\tilde{x} \sim \tilde{y}$ the set $A=\{n$ : $\left.x_{n} \neq y_{n}\right\}$ has density zero. Hence, we also have for every $\varepsilon>0$,

$$
\delta\left(\left\{n:\left|x_{n}-y_{n}\right| \geq \varepsilon\right\}\right)=0 .
$$

It means that $\varepsilon \in U^{s t}\left(\left|x_{n}-y_{n}\right|\right)$ and $\|\tilde{x}-\tilde{y}\|_{\infty}^{s t} \leq \varepsilon$. Since $\varepsilon$ is an arbitrary positive real number, then

$$
\|\tilde{x}-\tilde{y}\|_{\infty}^{s t}=0
$$

holds. The converse can be proven following same steps. So it is omitted.

Corollary 26. For all $\tilde{x}, \tilde{y} \in l_{\infty}^{s t},[\tilde{x}] \neq[\tilde{y}]$ if and only if $\|\tilde{x}-\tilde{y}\|_{\infty}^{s t} \neq 0$.

Remark 27. Let $\tilde{x} \in l_{\infty}^{s t}$ be an arbitrary element. If $\forall \tilde{y} \in[\tilde{x}]$, then there exists a set $K \subset \mathbb{N}$ such that $\delta(K)=1$ and $\left(\tilde{y}_{n}\right)_{n \in K}=\left(\tilde{x}_{n}\right)_{n \in K}$.

Proof. From Theorem 11 there exist $K_{1}, K_{2} \subset \mathbb{N}$ such that $\left(\tilde{x}_{n}\right)_{n \in K_{1}} \in l_{\infty}$ and $\left(\tilde{y}_{n}\right)_{n \in K_{2}} \in l_{\infty}$ hold. Since $\tilde{y} \in[\tilde{x}]$, then the set $\left\{n: x_{n} \neq y_{n}\right\}$ has density zero. So, $K_{3}:=\left\{n: x_{n}=y_{n}\right\} \subset \mathbb{N}$ has density 1 . If we consider $K:=K_{1} \cap K_{2} \cap K_{3}$, then it is clear that $\delta(K)=1$ and this gives that $(\tilde{x})_{n \in K}=(\tilde{y})_{n \in K}$ holds.

Remark 28. For $\tilde{z} \in l_{\infty}^{s t}$, let $\tilde{x}$ and $\tilde{y} \in[\tilde{z}]$. If there exist $\bar{x}$ and $\bar{y} \in l_{\infty}$ such that $\bar{x} \sim \tilde{x}$ and $\bar{y} \sim \tilde{y}$ hold, then $\bar{x} \sim \bar{y}$. 
Proof. Since $\tilde{x}, \tilde{y} \in[\tilde{z}]$, then $\tilde{x} \sim \tilde{z}$ and $\tilde{y} \sim \tilde{z}$. So, $\tilde{x} \sim \tilde{y}$ satisfied. Therefore, $\bar{x} \sim \tilde{x}, \tilde{x} \sim \tilde{y}$ and $\tilde{y} \sim \bar{y}$ gives that $\bar{x} \sim \bar{y}$.

For given $\tilde{x} \in l_{\infty}^{s t}$ define the set

$$
E(\tilde{x}):=\left\{\bar{x} \in l_{\infty}: \tilde{x} \sim \bar{x}\right\} .
$$

It is known that the set $s$ (set of all sequences) is a group under the usual sequence summation operation. For any given bounded sequences $\tilde{x}, \tilde{y}$ the sequence $\tilde{x}+(-\tilde{y})$ is also bounded. So, the space $l_{\infty}$ is subgroup of $s$.

To show $l_{\infty}^{s t}$ is actually a subgroup of $s$ we should ensure $l_{\infty}^{s t}$ is closed under summation operation and for any $\tilde{x} \in l_{\infty}^{s t}$ the sequence $-\tilde{x}$ is also belongs to $l_{\infty}^{s t}$. First statement is immediate a consequence of Lemma 24 and the second one is a consequence of Lemma 14. Hence $l_{\infty}^{s t}$ is subgroup of $s$.

Theorem 29. The quotient spaces $\tilde{l}_{\infty}^{s t}$ and $\tilde{l}_{\infty}$ are isomorphic.

Proof. Define a function $f$ between $\tilde{l}_{\infty}^{s t}$ and $\tilde{l}_{\infty}$ as follows:

$$
f: \tilde{l}_{\infty}^{s t} \rightarrow \tilde{l}_{\infty}
$$

where $f([\tilde{x}])=[\bar{x}]$ such that $\bar{x} \in E(\tilde{x})$.

To show $f$ is well-defined let us consider any two sequences $\tilde{a}$ and $\tilde{b}$ in $\tilde{l}_{\infty}^{s t}$. Then, there exist $\tilde{x}$ and $\tilde{y} \in l_{\infty}^{s t}$ such that $a=[\tilde{x}]$ and $b=[\tilde{y}]$ satisfied.

If $a=b$, then $[\tilde{x}]=[\tilde{y}]$ holds because $\sim$ is an equivalence relation on $s$. Hence, there exist $\bar{x} \in E(\tilde{x})$ and $\bar{y} \in E(\tilde{y})$ such that if $\bar{x} \sim \tilde{y}$ and $\bar{y} \sim \tilde{x}$ then $\bar{x} \sim \bar{y}$.

This means that $[\bar{x}]=[\bar{y}]$ implies that $f([\tilde{x}])=f([\tilde{y}])$, as a result of this we have $f(a)=f(b)$.

First implication follows from Theorem 11. Hence, $f$ is well-defined. It is clear that $f$ is an homomorphism of groups.

We claim that $f$ is also isomorphism. To show that $f$ is injective, for any $[\tilde{x}]$ and $[\tilde{y}] \in \tilde{l}_{\infty}^{s t}$. If $f([\tilde{x}])=f([\tilde{y}])$, then $[\bar{x}]=[\bar{y}]$. Therefore, $\bar{x} \sim \bar{y}$ and this gives that $\bar{y} \sim \tilde{x}$ and $\bar{x} \sim \tilde{y}$ from the definition of $f$ and transtivity of $\sim$. Which is implies that $\tilde{x} \sim \tilde{y}$ and hence $[\tilde{x}]=[\tilde{y}]$. Therefore, $f$ is an injective function.

Surjectivity of $f$ follows from Theorem 1 which implies that the set $E(\tilde{x})$ is always nonempty for every element of $l_{\infty}^{s t}$. Hence, there is always a sequence $\tilde{x}$ of $l_{\infty}^{s t}$ such that $\tilde{x}$ equivalent to some sequence $\bar{x}$ of $l_{\infty}$ with respect to relation $\sim$.

For any given sequence $\bar{x} \in l_{\infty}$, it can be constructed the mentioned sequence as follows:

$$
\tilde{x}_{n}:= \begin{cases}\bar{x}_{n}, & n \neq p_{i} \\ i, & n=p_{i}\end{cases}
$$

where $i \in \mathbb{N}$ and $p_{i}$ denotes the $i-t h$ prime number.

Then, it is clear that $\tilde{x} \sim \bar{x}$. Finally its convenient to say that $f$ is an isomorphism of groups.

So, the groups $l_{\infty}^{s t}$ and $l_{\infty}$ are isomorphic. 
Definition 30 (Convergence in $\left.\|.\|_{\infty}^{s t}\right)$. A sequence $\tilde{x}=\left(x_{n}\right)_{n \in \mathbb{N}} \in l_{\infty}^{\text {st }}$ is convergent to $x_{0}$ in the norm $\|.\|_{\infty}^{s t}$ if

holds.

$$
\left\|x_{n}-x_{0}\right\|_{\infty}^{s t}=0
$$

Definition 31 (Cauchy sequence in $\left.\|.\|_{\infty}^{s t}\right)$. A sequence $\tilde{x}=\left(x_{n}\right)_{n \in \mathbb{N}}$ is called Cauchy sequence in the norm $\|.\|_{\infty}^{s t}$ norm if

$$
\left\|x_{n}-x_{m}\right\|_{\infty}^{s t}=0
$$

holds.

Theorem 32. Let $\tilde{x}=\left(x_{n}\right) \in l_{\infty}^{\text {st }}$ be a sequence and $x_{0} \in \mathbb{R}$. Then, $\tilde{x}=\left(x_{n}\right)_{n \in \mathbb{N}}$ statistical convergent to $x_{0}$ if and only if $\tilde{x}=\left(x_{n}\right)_{n \in \mathbb{N}}$ convergent to $x_{0}$ in $\|.\|_{\infty}^{s t}$.

Proof. Assume that $\tilde{x}=\left(x_{n}\right)$ is convergent to $x_{0}$ statistically. Then, for every $\varepsilon>0$, the set $A(n, \varepsilon)=\left\{n:\left|x_{n}-x_{0}\right| \geq \varepsilon\right\}$ has density zero. That is,

$$
\delta(A(n, \varepsilon))=0
$$

holds. This implies that arbitrary $\varepsilon$ is a statistical upper bound of the sequence $\left(\left|x_{n}-x_{0}\right|\right)_{n \in \mathbb{N}}$. Hence,

$$
U^{s t}\left(\left|x_{n}-x_{0}\right|\right)=[\varepsilon, \infty)
$$

and this implies that

$$
\inf U^{s t}\left(\left|x_{n}-x_{0}\right|\right)=\varepsilon .
$$

since $\varepsilon>0$ is an arbitrary, then we have

$$
\left\|x_{n}-x_{0}\right\|_{\infty}^{s t}=0 .
$$

Now assume that $\left\|x_{n}-x_{0}\right\|_{\infty}^{s t}=0$ satisfied. So, $\inf U^{s t}\left(\left|x_{n}-x_{0}\right|\right)=0$ holds. It means that

$$
\delta\left(\left\{n:\left|x_{n}-x_{0}\right| \geq M\right\}\right)=0
$$

holds for every $M>0$. This is also true for any $\varepsilon>0$. Hence $\tilde{x}=\left(x_{n}\right)$ is statistical convergent to $x_{0}$.

Theorem 33. A sequence $\tilde{x}=\left(x_{n}\right) \in l_{\infty}$ is statistical Cauchy sequence if and only if $\tilde{x}=\left(x_{n}\right)$ is Cauchy sequence in $\|.\|_{\infty}^{s t}$.

Proof. Assume that $\tilde{x}=\left(x_{n}\right)$ is statistical Cauchy sequence. Then, for every $\varepsilon>0$, there exists $N \in \mathbb{N}$ such that any $n, m \geq N$ the set

$$
\left\{n:\left|x_{n}-x_{m}\right| \geq \varepsilon\right\}
$$

has density zero. That is;

$$
\delta\left(\left\{n:\left|x_{n}-x_{m}\right| \geq \varepsilon\right\}\right)=0 .
$$

This implies that arbitrary $\varepsilon$ is a statistical upper bound of the sequence $\left(\mid x_{n}-\right.$ $\left.x_{m} \mid\right)_{n, m \in \mathbb{N}}$. Hence, we have $U^{s t}\left(\left|x_{n}-x_{m}\right|\right)=[\varepsilon, \infty)$ and $\inf U^{s t}\left(\left|x_{n}-x_{m}\right|\right)=\varepsilon$. Since $\varepsilon$ is an arbitrary positive real number, then $\left\|x_{n}-x_{m}\right\|_{\infty}^{s t}=0$. 
Now assume that $\left\|x_{n}-x_{m}\right\|_{\infty}^{s t}=0$. From assumption $\inf U^{s t}\left(\left|x_{n}-x_{m}\right|\right)=0$ holds. This implies that for every $\varepsilon>0$

$$
\delta\left(\left\{n:\left|x_{n}-x_{m}\right|<\varepsilon\right\}\right)=0
$$

must be hold. Hence, $\tilde{x}=\left(x_{n}\right)$ is statistical Cauchy sequence.

Corollary 34. A number sequence $\tilde{x}=\left(x_{n}\right)$ convergent to $x_{0}$ in $\|.\|_{\infty}^{\text {st }}$ if and only if it is a Cauchy sequence in $\|.\|_{\infty}^{s t}$.

Theorem 35. Let $[\tilde{x}] \in \tilde{l}_{\infty}^{\text {st }}$ be an arbitrary equivalence class. If $\tilde{x}$ is statistical convergent to $x_{0} \in \mathbb{R}$, then all sequences $\tilde{y} \in[\tilde{x}]$ are statistical convergent to $x_{0}$.

Proof. Since $[\tilde{x}] \in \tilde{l}_{\infty}^{s t}$ is statistical convergent to $x_{0}$, then

$$
\left\|x_{n}-x_{0}\right\|_{\infty}^{s t}=0
$$

holds. Now, let $\tilde{y} \in[\tilde{x}]$ be an arbitrary sequence. It is also true that

$$
\|\tilde{x}-\tilde{y}\|_{\infty}^{s t}=0 .
$$

Following inequality

$$
\left\|\tilde{y}-x_{0}\right\|_{\infty}^{s t} \leq\|\tilde{y}-\tilde{x}\|_{\infty}^{s t}+\left\|\tilde{x}-x_{0}\right\|_{\infty}^{s t}
$$

with (4) and (5) gives that $\tilde{y}=\left(y_{n}\right)$ statistical convergent to $x_{0} \in \mathbb{R}$.

Corollary 36. If $\tilde{x} \in l_{\infty}^{s t}$ is not statistical convergent, then for all $\tilde{y} \in[\tilde{x}]$ are not statistical convergent.

In the following theorem, we will prove that the quotient space $\tilde{l}_{\infty}^{s t}$ is a Banach space with the norm produced by asymptotic density.

Without loosing generality we are going to use $\tilde{x}=\left(x_{n}\right)$ instead of equivalence class.

Theorem 37. $\left(\tilde{l}_{\infty}^{s t},\|\cdot\|_{\infty}^{s t}\right)$ is Banach space.

Proof. Since it is given in Theorem 20 (i) that statistical bounded sequence space $\tilde{l}_{\infty}^{s t}$ is already a linear vector space over real numbers, then we should verify only here $\left(\tilde{l}_{\infty}^{s t},\|\cdot\|_{\infty}^{s t}\right)$ is complete space.

For this purpose, let $\left(x^{n}\right) \in \tilde{l}_{\infty}^{s t}$ be an arbitrary Cauchy sequence in $\|.\|_{\infty}^{s t}$. Note that each element of $\left(x^{n}\right)$ is belongs to $l_{\infty}^{s t}$. So, the sequence $\left(x^{n}\right)$ can be express as

$$
\left(x^{n}\right)=\left(x_{1}^{n}, x_{2}^{n}, \ldots, x_{k}^{n}, \ldots\right),
$$

where $x_{k}^{n}=\left(x_{k}^{1}, x_{k}^{2}, \ldots\right)$ for all $n \in \mathbb{N}$. Since $\left(x^{n}\right)$ is Cauchy sequence with the norm $\|.\|_{\infty}^{s t}$, then there exists $N \in \mathbb{N}$ such that

$$
\left\|x^{n}-x^{m}\right\|_{\infty}^{s t}=0
$$

holds for all $n, m>N$. Thus,

$$
\inf U^{s t}\left(\left|x_{k}^{n}-x_{k}^{m}\right|\right)=0 .
$$


It means that for all $\varepsilon>0$,

$$
\delta\left(\left\{n:\left|x_{k}^{n}-x_{k}^{m}\right| \geq \varepsilon\right\}\right)=0
$$

holds. That is, for each $k$, the number sequences

$$
\left(x_{k}^{1}\right),\left(x_{k}^{2}\right), \ldots
$$

are statistical Cauchy sequence in $\mathbb{R}$. Since statistical Cauchy sequence is statistical convergent, then

$$
s t-\lim _{n \rightarrow \infty} x_{k}^{n}=x_{k}
$$

holds for all $k \in \mathbb{N}$.

Let $\tilde{x}=\left(x_{1}, x_{2}, \ldots\right)$ be a sequence consists of all statistical limits of the sequences $\left(x_{k}^{n}\right)$, respectively.

Now, we would like to show the sequence $\left(x^{n}\right)$ converges to $\tilde{x}=\left(x_{1}, x_{2}, \ldots\right)$ and belongs to the space $\tilde{l}_{\infty}^{s t}$.

From (6), for all $k \in \mathbb{N}$ and $\varepsilon>0$

$$
\delta\left(\left\{n:\left|x_{k}^{n}-x_{k}\right| \geq \varepsilon\right\}\right)=0
$$

holds. This gives that

$$
U^{s t}\left(\left|x^{n}-\tilde{x}\right|\right)=[\varepsilon, \infty)
$$

and

$$
\inf U^{s t}\left(\left|x^{n}-\tilde{x}\right|\right)=0
$$

hold because of $\varepsilon$ is an arbitrary number. This gives that $\left\|x^{n}-\tilde{x}\right\|_{\infty}^{s t}=0$. Therefore, the sequence $\left(x^{n}\right)$ converges to the $(\tilde{x})$ in $\|.\|_{\infty}^{s t}$.

Also, following inequality

$$
\|\tilde{x}\|_{\infty}^{s t} \leq\left\|\tilde{x}-x^{n}\right\|_{\infty}^{s t}+\left\|x^{n}\right\|_{\infty}^{s t}
$$

gives that $\tilde{x} \in \tilde{l}_{\infty}^{s t}$.

\section{Maximality of $l_{\infty}^{s t}$ And Nonporosity of $l_{\infty}$}

To prove $l_{\infty}^{s t}$ is maximal extension of the space $l_{\infty}$, we are going to follow the idea given by Fridy in 11 . Let's give the following lemma:

Lemma 38. If $\left(u_{n}\right)$ real valued sequence with $u_{n} \neq 0$ for infinitely many $n$, then there exists a sequence $\tilde{x}=\left(x_{n}\right) \in l_{\infty}^{\text {st }}$ such that

$$
\sum_{n=1}^{\infty} u_{n} x_{n}=\infty
$$

Proof. Let us consider increasing sequence $(n(k))$ of natural numbers such that

$$
n(k) \geq k^{2} \text { and } u_{n(k)} \neq 0
$$


are satisfied. Take into consider $\tilde{x}=\left(x_{n}\right)$ as

$$
x_{n}= \begin{cases}\frac{1}{u_{n(k)}}, & k=n(k) \\ 3, & k=n(k)+1 \\ 5, & \text { otherwise. }\end{cases}
$$

For $M=6$, following inclusion

$$
\left\{k:\left|x_{k}\right| \geq 6\right\} \subset\{n(k): k \in \mathbb{N}\}
$$

holds. Also, asymptotic density of the set $\left\{n^{2}: n \in \mathbb{N}\right\}$ is zero and this implies that $\delta(\{n(k): k \in \mathbb{N}\})=0$. It means that $\tilde{x} \in l_{\infty}^{s t}$. But, for the sequence $\tilde{x}$

$$
\sum_{n=1}^{\infty} u_{n} x_{n}=\sum_{k=1}^{\infty} u_{n(k)} x_{k}=\infty
$$

holds.

Let $T=\left(t_{n, k}\right)$ be any summability matrix. For any $\tilde{x}=\left(x_{k}\right) \in s$, if $(T \tilde{x})_{n}=$ $\left(\sum_{k=1}^{\infty} t_{n, k} x_{k}\right) \in l_{\infty}$, then $\tilde{x}$ is called $T$ bounded sequence. The set of all $T$-bounded sequences space is denoted by $l_{\infty}^{T}$ :

$$
l_{\infty}^{T}=\left\{\tilde{x}=\left(x_{k}\right) \in s: T(x)_{n} \in l_{\infty}\right\} .
$$

From Lemma 38 , in order to check a summability matrix include the statistical extension of $l_{\infty}$, it must be row-finite.

Theorem 39. There is no row finite matrix $T=\left(t_{n, k}\right)$ such that $l_{\infty}^{T}$ contains $l_{\infty}^{\text {st }}$.

Proof. Let $T=\left(t_{n, k}\right)$ which is any row finite summability matrix and constuct a sequence $\tilde{x}$ as follows:

Choose a nonzero entry, $t_{n(1), k^{\prime}(1)} \neq 0$. Then, choose $k(1) \geq k^{\prime}(1)$ such that $t_{n(1), k(1)} \neq 0$ and $t_{n(1), k}=0$ for all $k>k(1)$.

We can select an increasing sequence of row and column such that for each $m$,

$$
t_{n(m), k(m)} \neq 0, k(m) \geq m^{2}
$$

and

$$
t_{n(m), k}=0, \text { for all } k>k(m) .
$$

Now we can choose $\tilde{x}=\left(x_{k}\right)$ as:

$$
x_{k}= \begin{cases}\frac{1}{t_{n(m), k(m)}}\left[m-\sum_{i=0}^{m-1} t_{n(m), k(i)} x_{k(i)}\right], & k=k(m), \\ k^{2}, & k=k(m-1), \\ (-1)^{k}, & \text { otherwise. }\end{cases}
$$

Then, $\tilde{x}$ is not $T$-bounded sequence because

$$
(T x)_{n(m)}=(1,2, \ldots, m, . .) \notin l_{\infty} .
$$

But for any sufficiently large $M>0$ we have

$$
\left\{k:\left|x_{k}\right| \geq M\right\} \subset\{k(m), k(m-1): m \in \mathbb{N}\} .
$$


Since $\{k(m), k(m-1): m \in \mathbb{N}\} \subset\left\{m^{2}, m^{2}-1: m \in \mathbb{N}\right\}$, then it is a subset of

$$
\left\{m^{2}: m \in \mathbb{N}\right\} \cup\left\{m^{2}-1: m \in \mathbb{N}\right\} .
$$

So, we have $\tilde{x}=\left(x_{k}\right) \in l_{\infty}^{\text {st }}$ because

$$
\delta\left(\left\{m^{2}: m \in \mathbb{N}\right\} \cup\left\{m^{2}-1: m \in \mathbb{N}\right\}\right)=0 .
$$

Let us consider a function as :

$$
d_{\infty}^{s t}: \tilde{l}_{\infty}^{s t} \mathrm{x} \tilde{l}_{\infty}^{s t} \rightarrow[0, \infty)
$$

with

$$
d_{\infty}^{s t}(\tilde{x}, \tilde{y}):=\|\tilde{x}-\tilde{y}\|_{\infty}^{s t} .
$$

Since $\|.\|_{\infty}^{s t}$ is a norm on $\tilde{l}_{\infty}^{s t}$, then $d_{\infty}^{s t}$ is a metric on $l_{\infty}^{s t}$. So, we can consider $\left(\tilde{l}_{\infty}^{s t}, d_{\infty}^{s t}\right)$ as a metric space.

Definition 40. [17] A set $E$ in a Banach space $X$ (or in a general metric space $X)$ is called porous if there is $0<r<1$ such that for every $x \in E$ and every $\varepsilon>0$ there is a $y \in X$ with

$$
0<\operatorname{dist}(x, y)<\varepsilon
$$

and

$$
B(y, \operatorname{rdist}(x, y)) \cap E=\emptyset .
$$

In this case $r$ is porosity of $E$ in $X$. If such an $r$ is not exist then $E$ is called non-porous in $X$.

Notion of porosity of a set $E$ of a metric space $X$ at a point $x \in X$ concerns the size of "pores of $E$ " near to $x$. Now we want to investigate whether $l_{\infty}$ has pores in $l_{\infty}^{s t}$ or not.

Theorem 41. Bounded sequence space $l_{\infty}$ is non-porous subset of $l_{\infty}^{\text {st }}$.

Proof. Assume that $l_{\infty}$ is a porous subset of $l_{\infty}^{s t}$ with $r_{0} \in(0,1)$. That is, $\forall \tilde{x} \in l_{\infty}$ and $\varepsilon>0$, there exists $\tilde{y} \in l_{\infty}^{s t}$ such that $d_{\infty}^{s t}(\tilde{x}, \tilde{y})<\varepsilon$ and

$$
B\left(\tilde{y}, r_{0} \cdot d_{\infty}^{s t}(\tilde{x}, \tilde{y})\right) \cap l_{\infty}=\emptyset
$$

holds. From Theorem 11, there is a sequence $\bar{y} \in l_{\infty}$ such that $\tilde{y}=\bar{y}$ holds a.a.n.

Now, let's take a sequence $\bar{x}=\left(\bar{x}_{n}\right)$ as

$$
\bar{x}_{n}=\bar{y}_{n}+\frac{r_{0} \cdot d_{\infty}^{s t}(\tilde{x}, \tilde{y})}{2}
$$

for all $n \in \mathbb{N}$. Then,

$$
d_{\infty}^{s t}(\bar{x}, \bar{y})=\inf U^{s t}\left(\left|\overline{x_{n}}-\overline{y_{n}}\right|\right)=\frac{r_{0} \cdot d_{\infty}^{s t}(\tilde{x}, \tilde{y})}{2}
$$

This calculation gives that $\bar{x} \in B\left(\tilde{y}, r_{0} \cdot d_{\infty}^{s t}(\tilde{x}, \tilde{y})\right)$. This inclusion gives us a contradiction beacuse of (7). Hence, bounded sequence space $l_{\infty}$ is a non-porous subset of $l_{\infty}^{s t}$. 
Theorem 42. The sequence space $c_{s t}$ is non-porous in $l_{\infty}^{s t}$.

Proof. Assume that $c_{s t}$ is a porous subset of $l_{\infty}^{s t}$ with $r_{0} \in(0,1)$. Then, for all $\tilde{x}=\left(x_{n}\right) \in l_{\infty}^{s t}, \varepsilon>0$, there exists $\tilde{y} \in l_{\infty}^{s t}$ such that $d_{\infty}^{s t}(\tilde{x}, \tilde{y})<\varepsilon$ and

$$
B\left(\tilde{y}, r_{0} \cdot d_{\infty}^{s t}(\tilde{x}, \tilde{y})\right) \cap c_{s t} \neq \emptyset .
$$

Denote $r^{*}:=r_{0} \cdot d_{\infty}^{s t}(\tilde{x}, \tilde{y})$ and consider a sequence $\tilde{y}^{*}=\left(y_{n}^{*}\right)$ with $y_{n}^{*}=y_{n}+\frac{r^{*}}{2}$ for all $n \in \mathbb{N}$. It is clear that

$$
d_{\infty}^{s t}\left(\tilde{y}, \tilde{y}^{*}\right)=\inf U^{s t}\left(\mid y_{n}-y_{n}^{*}\right)=\inf U^{s t}\left(\frac{r^{*}}{2}\right)=\frac{r^{*}}{2}<r^{*}
$$

holds. Also $\tilde{y}^{*}=\left(y_{n}^{*}\right) \in l_{\infty}^{s t}$. From Theorem 12, $\left(\tilde{y}_{n}^{*}\right)$ can be represented as

$$
\left(\tilde{y}_{n}^{*}\right)=\left(t_{n}\right)+\left(z_{n}\right)
$$

such that $\left(t_{n}\right) \in l_{\infty}$. Bolzano-Weierstrass Theorem gives that $\left(t_{n}\right)$ has a convergent subsequence. Let us denote this sequence by $\left(t_{n}^{*}\right)$. Since every convergent sequence is statistical convergent, then $\left(t_{n}^{*}\right) \in c_{s t}$. Now we must show that $\left(t_{n}^{*}\right)$ belongs to $B\left(\tilde{y}, r^{*}\right)$. This holds from the fact

$$
d_{\infty}^{s t}\left(\tilde{y}, t_{n}^{*}\right)=\inf U^{s t}\left(\left|y_{n}-t_{n}^{*}\right|\right) \leq \inf U^{s t}\left(\mid y_{n}-y_{n}^{*}\right)<r^{*} .
$$

\section{CONClusion}

In accordance with the purpose of the study, $l_{\infty}^{s t}$ space covering $c_{s t}$ and $l_{\infty}$ spaces was built and it was shown that the space $l_{\infty}^{s t}$ is a lineer space and associated quotient space is a statistical Banach space. At the end of the paper also shown that $l_{\infty}^{s t}$ does not different from the spaces $l_{\infty}$ and $c_{s t}$ although it covers them.

The results obtained in the study can be generalized by considering different type of density on natural numbers such as logaritmic density, uniform density or density produced by any summability matrixmethod.

Is it possible to find an extension of the space $l_{\infty}$ such that equal to the space of all sequences? Or is there any extension of $l_{\infty}$ such that cardinality of complement of this extension in $s$ is finite or a most countable.

Let $T$ and $M$ be two regular matrixes. Under which conditions extensions of the space $l_{\infty}$ corresponding to $T$ and $M$ can be compared?

Authors Contribution Statement The authors contributed equally. All authors read and approved the final copy of the manuscript.

Declaration of Competing Interests The authors declare that they have no known competing financial interests or personal relationships that could have appeared to influence the work reported in this paper. 


\section{REFERENCES}

[1] Altınok M., Küçükaslan M., A-statistical convergence and A-statistical monotonicity, Applied Mathematics E-Notes, 13 (2013), 249-260.

[2] Altınok M., Küçükaslan M., Ideal limit superior-inferior, Gazi University Journal of Science, 30 (1) (2017), 401-411.

[3] Altınok M., Küçükaslan M., A-statistical supremum-infimum and A-statistical convergence, Azerbaijan Journal of Mathematics, 4 (2) (2014), 31-42.

[4] Altınok M., Porosity supremum-infimum and porosity convergence, Konuralp Journal of Mathematics, 6 (1) (2018), 163-170.

[5] Bilalov B., Nazarova T., On statistical convergence in metric spaces, Journal of Mathematics Research, 7 (1) (2015), 37-43.

[6] Bilalov B., Nazarova T., On statistical type convergence in uniform spaces, Bull. of the Iranian Math. Soc., 42 (4) (2016), 975-986.

[7] Cabrera M. O., Rosalsky A., Ünver M., Volodin A., On the concept of B-statistical uniform integrability of weighted sums of random variables and the law of large numbers with mean convergence in the statistical sense, TEST, (2020).

[8] Et M., Sengül H., Some Cesaro type summability spaces of order $\alpha$ and lacunary statistical convergence of order $\alpha$, Filomat, 28 (8) (2014), 1593-1602.

[9] Erdös P., Tenenboum G., Sur les densities de certaines suites dentiers, Proc. London Math. Soc., 59 (1989), 417-438.

[10] Fast H., Sur la convergence statistique, Colloquium Mathematicae, 2 (3-4) (1951), 241-244.

[11] Fridy J. A., On statistical convergence, Analysis, 5 (1985), 301-313.

[12] Fridy J. A., Khan M. K., Tauberian theorems via statistical convergnece, J.Math. Anal.Appl., 228 (1998), 73-95.

[13] Gadjiev A. D., Orhan C., Some approximation theorems via statistical convergnece, Rocky Mountain J. Math., 32 (2002), 129-138.

[14] Kaya E, Küçükaslan M., Wagner R., On statistical convergence and statistical monotonicity, Annales Univ. Sci. Budapest. Sect. Comp., 39 (2013), 257-270.

[15] Küçükaslan M., Altınok M., Statistical supremum-infimum and statistical convergence, The Aligarh Bulletin of Mathematics, 32 (1-2) (2013), 1-16.

[16] Küçükaslan M., Deger U., Dovgoshey O., On the Statistical Convergence of Metric-Valued Sequences, Ukrainian Mathematical Journal, 66 (5) (2014), 712-720.

[17] Lindenstrauss J., Preiss D., Tišer J., Fréchet Differentiability of Lipschitz Functions and Porous Sets in Banach Spaces, Princeton University Press, 41 William Street, Princeton, New Jersey, 2012.

[18] Lonetti P., Limit points of subsequences, Topology and its Applications, 263 (2019), 221-229.

[19] Miller H. I., A measure theoretical subsequence characterization of statistical convergence, Trans. Amer. Math. Soc., 347 (1995), 1881-1919.

[20] Pratulananda D., Savas E., On Generalized Statistical and Ideal Convergence of MetricValued Sequences, Ukrainian Mathematical Journal, 68 (1) (2017), 1849-1859.

[21] Salat T., On statistical convergent sequences of real numbers, Math. Slovaca, 30 (2) (1980), 139-150.

[22] Sanjoy Ghosal, K., Statistical convergence of a sequence of random variables and limits theorems, Applications of Mathematics, 58 (4) (2013), 423-437.

[23] Sandeep G., Bhardwaj V.K., On deferred $f$-statistical convergnece, Kyunpook Math.J., 58 (2018), 91-103.

[24] Steinhaus H., Sur la convergene ordinaire et la convergence asymptotique, Colloquium Mathematicae, 2 (3-4) (1951), 73-74.

[25] Zygmund A., Trigonometric series, vol II, Cambiridge Univ Press, 1979. 